\title{
ESTUDO DO LIQUOR DE CUPUAÇU ${ }^{1}$
}

\author{
Kelly de Oliveira COHEN ${ }^{2, *}$, Marisa de Nazaré Hoelz JACKIX ${ }^{3}$
}

\section{RESUMO}

O cupuaçu (Theobroma grandiflorum Schum), que é um fruto amazônico, vem conquistando a cada ano mais espaço no mercado nacional e internacional com a comercialização de sua polpa. Suas sementes, consideradas como subproduto, vem despertando interesse não só no mundo científico como pelas indústrias. Sabe-se que, a partir das sementes, pode-se obter produtos análogos aos oriundos das sementes de cacau, seguindo as mesmas etapas de processamento. Dentre esses produtos tem-se o liquor de cupuaçu, semelhante ao liquor de cacau que é um dos ingredientes da formulação do chocolate. Este trabalho teve como objetivo o estudo da obtenção e caracterização física, química e fisico-química do liquor de cupuaçu. Obteve-se três amostras de liquor de cupuaçu, cada qual partindo de lotes diferentes de fermentação. Foram avaliados a composição centesimal e o comportamento reológico. O liquor de cupuaçu apresentou alto teor de lipídios (63,93 a 66,51\%) e alto valor calórico $(677,35$ a $691,17 \mathrm{kcal} / 100 \mathrm{~g})$. O teor de proteínas variou de 8,95 a $10,31 \%$. A viscosidade plástica de Casson diminuiu com o aumento de temperatura. A distribuição do tamanho das partículas influenciou nas diferenças de viscosidades entre as amostras, havendo maior redução de viscosidade plástica com o aumento da temperatura nas amostras que apresentaram maior diâmetro de partículas. A gordura de cupuaçu apresentou comportamento newtoniano à $40^{\circ} \mathrm{C}$. Palavras-chave: chocolate; Theobroma grandiflorum; gordura de cupuaçu.

\section{SUMMARY}

STUDY OF CUPUASSU LIQUOR. Cupuassu (Theobroma grandiflorum Schum) is an amazon fruit, that each year is conquering more space in national and international market with the commercialization of its pulp. Its seeds, considered as subproducts, are arousing interest not only in the scientific world but also in the industry. It's known that from those seeds, it can be obtained analogous products as those cocoa seeds, following the same stages of processing. Among those products there is the cupuassu liquor, similar to cocoa liquor that is one of the ingredients of the chocolate formulation. The objective of this work was to study the physical, chemical and physicochemical acquisition and characterization of cupuassu liquor. Three samples of cupaussu liquor were obtained, each one coming from a fermentation lot. Each lot was subjected to centesimal composition and rheological study. The cupuassu liquor presented high content of fat (63.93 to $66.51 \%)$ and caloric value $(677.35$ to $691.17 \mathrm{kcal} / 100 \mathrm{~g})$. Its protein content varied from 8.95 to $10.31 \%$. Its Casson plastic viscosity diminished as the temperature increased. The distribuition of the particles sizes influenced in the viscosity differences among samples. A higher reduction of plastic viscosity was found with the increase of temperature in the samples that presented bigger participles diameter. The cupuassu fat presented Newtonian behavior at $40^{\circ} \mathrm{C}$.

Keywords: chocolate; Theobroma granfiflorum; cupuassu fat.

\section{1 - INTRODUÇÃO}

O cupuaçu é um dos mais importantes frutos tipicamente amazônicos. Seu valor econômico encontrase na polpa, que é consumida na forma de suco, néctar, iogurte, sorvete, creme, licor, torta, geléia, compota, biscoito, sorvete, e outros doces, os quais, na sua maioria, são processados de forma artesanal, em pequenas escalas de produção.

A produção de frutos de cupuaçu no Estado do Pará foi de 9.737 milhões em 1998, aumentando para 15.881 milhões, em 1999, e 21.479 milhões, em 2000 [21]. Além do crescimento na produção de frutos, cresce também a industrialização de sua polpa, que é comercializada nos Estados produtores de cupuaçu (Pará, Amazonas, Acre e Rondônia) e em outros Estados do Brasil e no exterior.

\footnotetext{
1. Recebido para publicação em 13/05/2004. Aceito para publicação em 17/12/2004 (001344).

2. EMBRAPA Amazônia Oriental. Trav. Dr. Enéas Pinheiro s/n, Caixa Postal 48, CEP 66095-100, Belém-PA. E-mail: cohen@cpatu.embrapa.br 3. Departamento de Tecnologia de Alimentos - Faculdade de Engenharia Alimentos - Universidade Estadual de Campinas (UNICAMP).

* A quem a correspondência deve ser enviada.
}

O aumento da industrialização da polpa de cupuaçu, que já é comercializada em outras regiões do Brasil e no exterior, tem propiciado volume significativo de sementes, que correspondem a $20 \%$ do peso do fruto. Estas são ricas em gordura e, quando fermentadas, secas e torradas adequadamente, podem ser utilizadas na elaboração de produtos análogos aos oriundos das sementes de cacau, utilizando as mesmas etapas de processamento, devendo-se ajustar os parâmetros dos processos envolvidos.

Dentre os produtos que podem ser obtidos das sementes de cupuaçu tem-se o liquor de cupuaçu, semelhante ao liquor de cacau que é definido, segundo FANG et al. [9], como uma dispersão de partículas de cacau envolvidas por uma fase gordurosa contínua composta pela manteiga de cacau. O liquor de cupuaçu pode ser empregado na formulação de produtos análogos ao chocolate, bolos, biscoitos e sorvetes, podendo-se extrair a gordura de cupuaçu, que é objeto de pesquisa nas indústrias alimentícia e farmacêutica.

COHEN [6] estudou a aplicação de liquor de cupuaçu em duas formulações de produto análogo ao chocolate ao leite: uma com 50\% de substituição de liquor de cacau por liquor de cupuaçu e 50\% de substituição de manteiga de cacau por gordura de cupuaçu (formulação $\mathrm{F}_{50}$ ) e a segunda com $100 \%$ de substituição desses mes- 
mos ingredientes (formulação $\mathrm{F}_{100}$ ). Por análise sensorial, verificou-se que a formulação $F_{50}$ apresentou notas superiores (escala estruturada de 9 pontos) para os atributos cor $(8,28)$, aroma $(7,53)$, sabor $(7,68)$ e aceitação global $(7,78)$ em relação a formulação $F_{100}$ (cor= 6,50 , aroma $=6,98$, sabor $=6,90$ e aceitação global $=$ $6,78)$, ficando esta formulação com nota superior àquela somente no atributo textura, que foi de 7,95 contra 7,65 da formulação $F_{50}$. Para a atitude de compra do consumidor, numa escala de 1 (eu certamente não compraria este produto) a 5 (eu certamente compraria este produto), a nota para a formulação $F_{50}$ foi de 4,20 , enquanto a da formulação $\mathrm{F}_{100}$ foi de 3,45. Diante desses resultados, o autor concluiu que a substituição de $50 \%$ de liquor e manteiga de cacau por liquor e gordura de cupuaçu na formulação do chocolate, tem futuro promissor em sua comercialização.

Para a aplicação industrial do liquor de cupuaçu na elaboração de produtos alimentícios, há a necessidade de se estudar seu processo de fabricação, bem como a determinação de suas características físicas, químicas e físico-químicas, que constituem o objetivo deste trabalho.

\section{2 - MATERIAL E MÉTODOS}

\section{1 - Material}

As sementes de cupuaçu foram coletadas, fermentadas e secas na Cooperativa Agrícola Mista de ToméAçu (CAMTA), localizada em Tomé-Açu, Estado do Pará.

\section{2 - Métodos}

\subsection{1 - Processo de obtenção de sementes de cupuaçu}

Para a obtenção de sementes de cupuaçu foram utilizados cerca de $600 \mathrm{~kg}$ de fruto para cada lote a ser fermentado (lotes F1, F2 e F3). Após a quebra dos frutos, com o auxílio de cutelo, suas sementes foram retiradas para serem despolpadas mecanicamente, deixando aderidas a esta cerca de $5 \%$ de polpa para a realização da etapa de fermentação.

\subsection{2 - Fermentação e secagem de sementes de cupuaçu}

As sementes de cupuaçu foram fermentadas em caixa de madeira, construída de acordo com GRIMALDI [13], com dimensões de $190 \mathrm{~cm}$ de comprimento, $120 \mathrm{~cm}$ de largura e $60 \mathrm{~cm}$ de altura e espaço entre as tábuas de fundo de $0,2 \mathrm{~cm}$ para o escoamento dos líquidos gerados durante o processo fermentativo. A caixa possui três compartimentos com forma e volume adequados para o processo e com capacidade para $160 \mathrm{~kg}$ de sementes, devendo ser colocada sob abrigo de chuva e sol.

Inicialmente, as sementes foram colocadas no primeiro compartimento da caixa, juntamente com folhas picadas de bananeira para proporcionar a inoculação dos microrganismos existentes na superficie dessas folhas, e cobertas com sacos de aniagem para auxiliar a retenção de calor gerado durante a fermentação. $\mathrm{O}$ tempo total do processo foi de 7 dias, com revolvimento das sementes após $48 \mathrm{~h}$ para o $2^{\circ}$ compartimento e $72 \mathrm{~h}$ para o $3^{\circ}$ compartimento da caixa. Foram determinadas as temperaturas diárias da massa em diferentes niveis (superfície, meio e fundo), com o auxílio de um termômetro de mercúrio.

Após o processo fermentativo, as sementes foram secas ao sol, em barcaça de madeira, durante 13 dias, até obtenção de umidade residual de aproximadamente $6 \%$, determinada com o auxílio de medidor de umidade de cacau.

\subsection{3 - Prova de corte}

A qualidade do processo fermentativo das sementes de cupuaçu foi avaliada pela prova de corte, conforme o método proposto para o cacau, Resolução n ${ }^{\circ} 42$ do CONSELHO NACIONAL DE COMÉRCIO EXTERIOR [7].

Foram retiradas, aleatoriamente, de cada lote, 100 amêndoas, as quais foram seccionadas de forma longitudinal, e observadas uma a uma. Tal procedimento foi realizado em triplicata.

\subsection{4 - Processo de obtenção dos nibs de cupuaçu}

As amêndoas de cupuaçu foram quebradas em moinho de facas, obtendo-se uma mistura de nibs (fragmentos dos cotilédones), cascas, nibs aderidos às cascas e amêndoas. Essa mistura foi submetida ao processo de peneiração, utilizando um granutest com peneiras de aberturas de 4, 9,51, 11,2 e 22,6mm. O material que passou através da peneira de $4 \mathrm{~mm}$ foi descartado e o material retido nas demais peneiras (nibs, nibs aderidos às cascas, cascas e amêndoas) passou por processo de separação manual, sendo que os nibs aderidos às cascas foram separados destas com auxílio de faca. Foram considerados os nibs com tamanhos de 4 a $10 \mathrm{~mm}$.

\subsection{5 - Processo de obtenção do liquor de cu- puaçu}

Primeiramente, os nibs de cupuaçu foram torrados em torrador elétrico rotativo, em lotes de $180 \mathrm{~g}$, à temperatura de $150^{\circ} \mathrm{C}$ por 40 minutos. Em seguida, os nibs já torrados passaram por processo de moagem, para a obtenção das amostras de liquor de cupuaçu (LF1, LF2 e LF3). Estes foram refinados em refinador PILON, composto de três cilindros horizontais de aço inoxidável, encamisados e resfriados com fluido refrigerante (água e álcool). Para cada lote, o processo foi conduzido 4 vezes, reduzindo-se a cada passagem a distância entre os cilindros.

\subsection{6 - Caracterização física, química e físico- quimica das amostras de liquor de cupuaçu}

- Teor de umidade - método 31.1.02, da AOAC [1].

- Teor de gordura - método 31.4.02, da AOAC [1]. 
- Teor de proteínas - método 31.1.08, da AOAC [1].

- Teor de fibras totais - método Acid Detergent Fibre (ADF), segundo GOERING \& VAN SOEST [12].

- Teor de cinzas - método 31.1.04, da AOAC [1].

- Teor de carboidratos totais - calculado por diferença.

- Valor calórico total - calculado segundo KALIL [15], PASSMORE, NICOL \& RAO [20] e USDA [24].

- $\quad \mathrm{pH}$ - método 31.1.07, da AOAC [1].

- Acidez total titulável - método 11.14.3, da AOAC [1].

- Cor - determinada em Espectrofotômetro HUNTERLAB/COLORQUEST II. A leitura foi realizada com o aparelho ajustado em reflectância, com especular incluída, utilizando-se o padrão de calibração branco (n C6299 de 03/96) e preto ( ${ }^{\circ}$ C6299G de 03/96). A configuração incluiu iluminante D65 e ângulo $10^{\circ}$. As leituras foram realizadas em sistema CIELAB $\left(L^{*}, a^{*}\right.$ e $\left.b^{*}\right)$ a temperatura de $22^{\circ} \mathrm{C}$.

- Distribuição do tamanho das partículas - as amostras de liquor de cupuaçu foram desengorduradas com solvente orgânico (éter de petróleo) e secas para, posteriormente, ser realizada a determinação da distribuição do tamanho das partículas, que foi realizado em fotosedimetrômetro LUMOSED. Os resultados foram obtidos com o auxílio do software GRANUTEST. Utilizou-se como fluido sedimentador a acetona PA.

- Propriedades reológicas - determinadas em reômetro programável BROOKFIELD (modelo RVDVIII), utilizando programa desenvolvido por GILABERT-ESCRIVÁ [10] com spindle \# 15, na temperatura de $40^{\circ} \mathrm{C}$ (Tabela 1$)$.

TABELA 1. Programa desenvolvido em reômetro programável BROOKFIELD para as medições da viscosidade plástica de Casson e do limite de escoamento de amostras de liquor.

\begin{tabular}{cccc}
\hline Tempo (s) & Rotação (rpm) & Tempo (s) & Rotação (rpm) \\
\hline$* 180$ & 5 & 15 & 20 \\
$* * 180$ & 50 & 15 & 30 \\
3 & 125 & 10 & 40 \\
3 & 100 & 6 & 50 \\
6 & 50 & 6 & 60 \\
15 & 20 & 5 & 70 \\
30 & 10 & 5 & 80 \\
60 & 5 & 4 & 90 \\
45 & 7,5 & 3 & 100 \\
30 & 10 & 3 & 110 \\
25 & 15 & 3 & 125
\end{tabular}

Fonte: GILABERT-ESCRIVÁ [10].

*Homogeneização da temperatura da amostra.

**Pré-cisalhamento da amostra.

\subsection{7 - Processo de obtenção e análises físicas e químicas da gordura de cupuaçu}

- Extração - para a extração da gordura de cupuaçu se utilizou prensa hidráulica com capacidade para $60 \mathrm{kgf} / \mathrm{cm}^{2}$. As amostras de liquor de cupuaçu foram aquecidas em forno de microondas até a temperatura de $80^{\circ} \mathrm{C}$, acondicionadas em sacos de lona e colocadas dentro do cilindro da prensa. O tempo total de extração foi de 40 minutos, sendo que nos primeiros 5 minutos utilizou-se pressão de $10 \mathrm{kgf} / \mathrm{cm}^{2}$, em seguida aumentou-se para 20kgf/ $\mathrm{cm}^{2}$ por mais 5 minutos, $30 \mathrm{kgf} / \mathrm{cm}^{2}$ por mais $5 \mathrm{mi}-$ nutos e, finalmente, $40 \mathrm{kgf} / \mathrm{cm}^{2}$ no tempo restante $(25$ minutos). Tal procedimento é necessário para que os sacos de lona não sofram ruptura. Após a extração das gorduras, estas foram filtradas para a retirada de possiveis partículas sólidas provenientes das amostras de liquor.

- Composição de ácidos graxos - realizada por cromatografia gasosa (CG) de ésteres metilicos, segundo HARTMAN \& LAGO [14], seguindo o método oficial da AOCS Ce 1-62 [2].

- Composição de triacilgliceróis - realizada em cromatografia líquida de alta eficiência (HPLC) em sistema de fase reversa com detector de índice de refração pelo método AOCS Ce 5b-89 [2].

- Viscosidade e limite de escoamento - determinada em reômetro programável BROOKFIELD (modelo RVDVIII), com spindle \#18, utilizando como temperatura de medição $40^{\circ} \mathrm{C}$, seguindo o programa da Tabela 2.

TABELA 2. Programa desenvolvido em reômetro programável BROOKFIELD para as medições de viscosidade das amostras de gordura de cupuaçu.

\begin{tabular}{lccccc}
\hline Rotaçăo (rpm) & 210 & 220 & 230 & 240 & 250 \\
\hline Tempo $(\min )$ & 1 & 1 & 1 & 1 & 1 \\
\hline
\end{tabular}

\section{RESULTADOS E DISCUSSÃo}

\section{1. - Perfil de temperatura durante processo fermentativo}

Na Figura 1 apresentam-se os perfis de temperatura durante o processo fermentativo de três lotes de sementes de cupuaçu (F1, F2 e F3).

Observa-se pela Figura 1 que para os lotes F2 e F3 as temperaturas máximas obtidas na fermentação foram de $47^{\circ} \mathrm{C}$ e $49^{\circ} \mathrm{C}$, respectivamente, alcançadas no terceiro dia do processo. Tais lotes apresentaram perfil de temperatura com comportamentos semelhantes, diferenciando somente do quinto para o sétimo dia de fermentação. Tais oscilações ocorrem devido às atividades metabólicas dos microrganismos. Entretanto, não houve comprometimento na qualidade dos produtos, como será visto no item 3.2. 
Para boa fermentação comercial de cacau, a temperatura da massa de semente deve atingir $45^{\circ} \mathrm{C}$ a $50^{\circ} \mathrm{C}$ em aproximadamente 3 dias, devendo-se manter tais valores até o término do processo [22]. Segundo VASCONCELOS [25], com as sementes de cupuaçu ocorre o mesmo, pois ao fermentar as sementes de cupuaçu em caixa T-60, após 3 dias a temperatura da massa foi de $48^{\circ} \mathrm{C}$, sendo esta a temperatura máxima alcançada durante os 7 dias da fermentação, baixando para $43^{\circ} \mathrm{C}$ e $45^{\circ} \mathrm{C}$ em dias posteriores e mantendo-se assim até o término do processo.
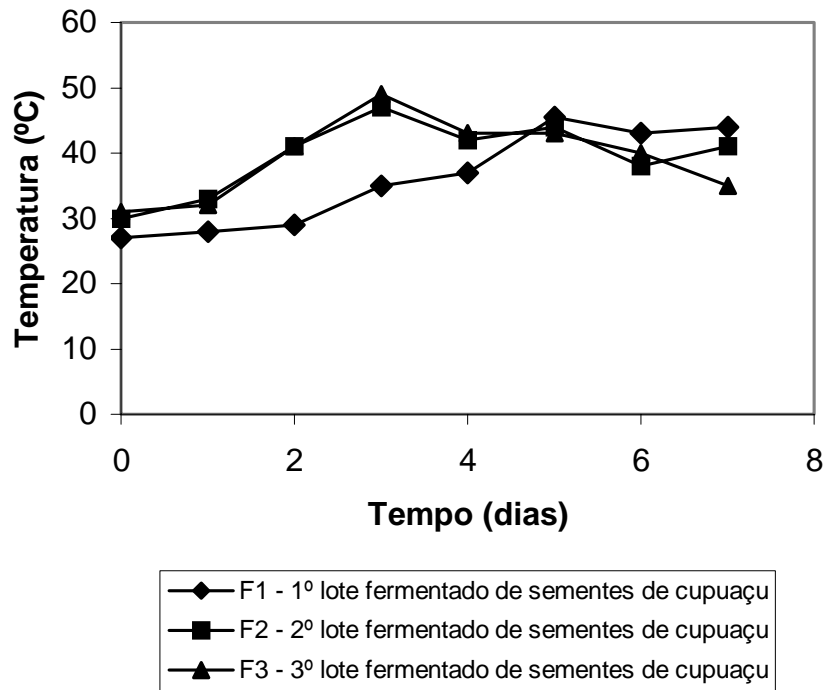

FIGURA 1. Perfil de temperatura durante o processo fermentativo das sementes de cupuaçu.

O lote F1 apresentou perfil de temperatura atípico, visto que no terceiro dia do processo, quando se espera considerável aumento de temperatura, esta foi de somente $35^{\circ} \mathrm{C}$, alcançando a temperatura máxima no quinto dia, comprometendo o processo fermentativo. Isso ocorreu devido às condições climáticas no dia do recebimento das sementes deste lote, pois como chovia bastante no local, as sementes encontravam-se molhadas, prejudicando a atividade metabólica dos microrganismos.

Segundo DIAS [8], o aumento de temperatura da massa durante a fermentação é atribuido à atividade metabólica dos microrganismos. Durante o primeiro dia, predominam certas espécies de leveduras que exigem pouco oxigênio, e a temperatura não é tão elevada. Com o consumo de ácido cítrico pelas leveduras, o valor do $\mathrm{pH}$ da polpa aumenta gradativamente, tornando o meio mais favorável para bactérias produtoras de ácido lático. Quando o pH da polpa atinge valores acima de 4,0 começam a predominar bactérias produtoras de ácido acético, alcançando temperaturas mais elevadas, em torno de 45 a $50^{\circ} \mathrm{C}$.

\section{2 - Classificação das sementes fermentadas e se- cas de cupuaçu}

$\mathrm{Na}$ Tabela 3 apresentam-se os resultados da prova de corte realizada nas sementes fermentadas e secas de cupuaçu.
TABELA 3. Classificação das sementes fermentadas e secas (amêndoa) de cupuaçu.

\begin{tabular}{ccccc}
\hline & \multicolumn{4}{c}{ Prova de corte } \\
\cline { 2 - 5 } Amostra & ${ }^{*} \mathrm{BF}(\%)$ & ${ }^{*} \mathrm{PF}(\%)$ & ${ }^{*} \mathrm{MF}(\%)$ & *Defeitos (\%) \\
\hline F1 & - & $49,0 \pm 3,6$ & $49,3 \pm 3,2$ & $1,7 \pm 0,6$ \\
F2 & $67,7 \pm 2,5$ & $20,3 \pm 2,1$ & $10,7 \pm 1,5$ & $1,3 \pm 0,6$ \\
F3 & $41,6 \pm 5,2$ & $35,6 \pm 6,5$ & $21,5 \pm 3,0$ & $1,3 \pm 1,2$ \\
\hline
\end{tabular}

*Média de três medições.

$\mathrm{F} 1-1^{\circ}$ lote fermentado de sementes de cupuaçu.

F2 - $2^{\circ}$ lote fermentado de sementes de cupuaçu.

$\mathrm{BF}$ - bem fermentadas / PF - parcialmente fermentadas / MF - mal fermentadas.

Uma amêndoa de cacau bem fermentada apresenta cotilédone de coloração marrom e uma mal fermentada de coloração violeta a púrpura, em grande parte de sua extensão. Particularmente, com relação à amêndoa de cupuaçu, esta apresenta coloração marrom claro quando mal fermentada e marrom avermelhado quando bem fermentada, além da presença de sulcos pronunciados em seu cotilédone, pois a ausência destes caracteriza uma amêndoa como parcialmente fermentada.

De acordo com a Resolução No 42 do CONCEX [7], para que as sementes fermentadas e secas de cacau apresentem classificação do Tipo I (superior), a soma dos defeitos não deve ultrapassar a tolerância de $6 \%$, sendo $2 \%$ para cada defeito isoladamente. Seguindo a mesma orientação para o cupuaçu, os três lotes de sementes fermentadas e secas (F1, F2 e F3) foram classificadas como Tipo I (Tabela 3). Entretanto, verifica-se que no lote $\mathrm{F} 1$ não foram encontradas sementes bem fermentadas $(\mathrm{BF})$, somente parcialmente $(\mathrm{PF})$ e mal fermentadas $(\mathrm{MF})$, confirmando o que foi visto no item 3.1 , ou seja, que o processo fermentativo deste lote não foi devidamente conduzido.

Todos os lotes de sementes fermentadas e secas (F1, F2 e F3) apresentaram aspecto externo bom e aroma típico livre de odores estranhos que podem ser incorporados na etapa de secagem.

\section{3 - Rendimento do processo de descascamento das amêndoas de cupuaçu}

$\mathrm{Na}$ Tabela 4 encontram-se os rendimentos de nibs de cupuaçu, obtidos por processo de descascamento.

TABELA 4. Rendimento do processo de descascamento das amêndoas de cupuaçu.

\begin{tabular}{cc}
\hline${ }^{*}$ Amostra & ${ }^{* *}$ Rendimento (\%) \\
\hline NF1 & 48,1 \\
NF2 & 49,7 \\
NF3 & 52,5 \\
\hline
\end{tabular}

*Nibs com tamanhos de $4 \mathrm{~mm}$ a $10 \mathrm{~mm}$.

**Rendimento calculado como a porcentagem de nibs em relação ao peso total das amêndoas.

NF1 - nibs de cupuaçu provenientes do lote F1 de sementes fermentadas e secas

NF2 - nibs de cupuaçu provenientes do lote F2 de sementes fermentadas e secas de

NF3 - nibs de cupuaçu provenientes do lote F3 de sementes fermentadas e secas de cupuaçu. 
No processo de descascamento das amêndoas de cacau, após a passagem destas pelo moinho, utiliza-se coluna de ar para separar as cascas dos nibs. Entretanto, para o cupuaçu tal procedimento não é viável, pois este apresenta casca mais espessa e pesada e cotilédone mais frágil que os do cacau. Logo, ao se utilizar esse equipamento para o cupuaçu, seus nibs são arrastados pela coluna de ar, ocasionando queda em seu rendimento.

LOPES, GARCIA \& VASCONCELOS [17] utilizaram em seu processo de obtenção de nibs de cupuaçu coluna de ar de separação de casca e obteve rendimento $(34,7 \%)$ significativamente inferior aos obtidos neste trabalho $(48,1$ a $52,5 \%)$.

\section{4 - Rendimento do processo de torração}

Na Tabela 5 verificam-se os rendimentos do processo de torração dos nibs de cupuaçu.

TABELA 5. Rendimento do processo de torração dos nibs de cupuaçu.

\begin{tabular}{cc}
\hline Amostra & *Rendimento (\%) \\
\hline TF1 & 75,1 \\
TF2 & 69,6 \\
TF3 & 79,0
\end{tabular}

*Rendimento calculado como a porcentagem de nibs torrados em relação ao peso total dos nibs não torrados.

$\mathrm{TF} 1$ - nibs torrados de cupuaçu provenientes do lote F1 de sementes fermentadas e secas de cupuaçu.

TF2 - nibs torrados de cupuaçu provenientes do lote F2 de sementes fermentadas

e secas de cupuaçu.
TF3 - nibs torrados de cupuaçu provenientes do lote F3 de sementes fermentadas e secas de cupuaçu.

A torração é um tratamento térmico cujo objetivo principal é a conversão dos precursores do sabor de chocolate (aminoácidos livres e açúcares redutores), desenvolvidos durante a fermentação, a sabor. Os métodos mais comuns de torração são aqueles realizados nas amêndoas inteiras e nos nibs. Neste trabalho, optou-se por realizar o processo de torração dos nibs para proporcionar torração mais homogênea.

Durante a torração ocorre mudança na textura do cotilédone deixando-o mais quebradiço. Como os nibs de cupuaçu são frágeis, ocorreu sua intensa fragmentação devido a essa mudança na textura, ajudada pela movimentação do tambor do torrador, ocasionando perda parcial, ficando o seu rendimento na faixa de 69,6 a $79,0 \%$.

\section{5 - Rendimento dos processos de moagem e de refino}

Na Tabela 6 verificam-se os rendimentos dos processos de moagem dos nibs torrados e do refino das amostras de liquor.

Os processos de moagem e de refino apresentaram ótimos rendimentos (Tabela 6). Ao passar o material no refinador, este se apresentou na forma de um filme que foi raspado formando flocos finos. As amostras de liquor de cupuaçu (LF1, LF2 e LF3) apresentaram aroma e aparência característicos e semelhantes ao liquor de cacau.

TABELA 6. Rendimento dos processos de moagem dos nibs torrados de cupuaçu e do refino das amostras de liquor de cupuaçu.

\begin{tabular}{ccc}
\hline Amostra & $\begin{array}{c}\text { *Rendimento (\%) } \\
\text { do processo de moagem }\end{array}$ & $\begin{array}{c}\text { **Rendimento (\%) } \\
\text { do processo de refino }\end{array}$ \\
\hline LF1 & 99,9 & 98,7 \\
LF2 & 99,8 & 97,8 \\
LF3 & 99,5 & 97,5
\end{tabular}

* Rendimento calculado como a porcentagem de nibs torrados e moídos em

relação ao peso total dos nibs torrados.

LF1 - liquor de cupuaçu proveniente do lote F1 de sementes fermentadas e secas de cupuaçu.

LF3 - liquor de cupuaçu proveniente do lote F3 de sementes fermentadas e secas de cupuaçu.

3.6 - Resultados obtidos nas análises físicas, químicas e físico-quimicas das amostras de liquor de cupuaçu

\subsection{1 - Composição físico-quimica das amostras de liquor de cupuaçu}

Na Tabela 7 encontra-se a composição físico-química das amostras de liquor de cupuaçu.

TABELA 7. Composição físico-química, em base seca, e teor de umidade e valor calórico das amostras de liquor de cupuaçu.

\begin{tabular}{lccc}
\hline & \multicolumn{3}{c}{ Amostra } \\
\hline & LF1 & LF2 & LF3 \\
\cline { 2 - 4 } Umidade (\%) & $0,97 \pm 0,03^{\mathrm{a}}$ & $0,72 \pm 0,03^{\mathrm{c}}$ & $0,80 \pm 0,06^{\mathrm{b}}$ \\
Proteínas (\%) & $10,31 \pm 0,07^{\mathrm{a}}$ & $8,95 \pm 0,14^{\mathrm{c}}$ & $9,27 \pm 0,04^{\mathrm{b}}$ \\
Lipídios (\%) & $63,93 \pm 0,06^{\mathrm{c}}$ & $66,51 \pm 0,44^{\mathrm{a}}$ & $65,77 \pm 0,12^{\mathrm{b}}$ \\
Fibras (\%) & $7,84 \pm 0,52^{\mathrm{a}}$ & $8,20 \pm 0,62^{\mathrm{a}}$ & $7,14 \pm 0,16^{\mathrm{a}}$ \\
Cinzas (\%) & $2,74 \pm 0,04^{\mathrm{a}}$ & $2,28 \pm 0,01^{\mathrm{b}}$ & $2,28 \pm 0,02^{\mathrm{b}}$ \\
Carboidratos totais (\%) & $15,18^{\mathrm{a}}$ & $14,07^{\mathrm{a}}$ & $15,54^{\mathrm{a}}$ \\
Energia (kcal/100g) & $677,35^{\mathrm{b}}$ & $690,63^{\mathrm{a}}$ & $691,17^{\mathrm{a}}$ \\
\hline
\end{tabular}

Os valores de uma mesma linha, com a mesma letra, não diferem significativamente entre si (Teste de Tukey a 5\% de significância). Testes realizados em

triplicata. de cupuaçu.

LF2 - liquor de cupuaçu proveniente do lote F2 de sementes fermentadas e secas

LF3 - liquor de cupuaçu proveniente do lote F3 de sementes fermentadas e secas de cupuaçu.

Com relação ao conteúdo de umidade, as amostras de liquor de cupuaçu (LF1, LF2 e LF3) apresentaram valores inferiores a $1 \%$, abaixo do recomendado pela litera- 
tura (em torno de 1\%), segundo TANERI [23]. O principal motivo pelo baixo conteúdo de umidade do liquor é devido ao processo de torração, que reduz a umidade de, aproximadamente, $8 \%$ para $2 \%$ ou abaixo desta. Um alto conteúdo de umidade no liquor pode ocasionar aumento de sua viscosidade plástica, acarretando problemas no processamento de produtos elaborados com o mesmo.

Comparando-se estatisticamente as amostras de liquor de cupuaçu entre si (Tabela 7), verifica-se que não houve diferença ao nivel de 5\% de significância nos teores de fibras e de carboidratos totais. No que se refere a proteínas e lipídios, houve diferença entre as três amostras. LF1 diferiu significativamente no teor de cinzas e valor energético de LF2 e LF3.

$\mathrm{O}$ alto teor de lipídios encontrado nas amostras de liquor de cupuaçu (63,93 a 66,51\%) é de grande interesse comercial. Atualmente, uma pequena fração desta gordura começa a ter aplicação na indústria de cosméticos. Porém, uma aplicação mais nobre e promissora está na utilização em alimentos.

LUCCAS [19] estudou o fracionamento térmico e a obtenção de gorduras de cupuaçu alternativas à manteiga de cacau na fabricação de chocolates e concluiu que a estearina de cupuaçu, obtida a $30^{\circ} \mathrm{C}$ na velocidade de 20rpm, pode ser utilizada como alternativa à manteiga de cacau, podendo ser aplicada com sucesso na fabricação de chocolates amargo, ao leite e branco, contribuindo com até $5 \%$ com base no peso total da formulação, sem alterar as características físicas e sensoriais do produto.

GILABERT-ESCRIVÁ [11] ao estudar a aplicação de gordura zero-trans pura e desodorizada de cupuaçu em panificação, concluiu que tanto a gordura de cupuaçu pura como à desodorizada podem ser usadas em substituição à gordura hidrogenada na elaboração de bolos. $\mathrm{O}$ autor verificou que a gordura de cupuaçu tem vantagem sobre a gordura hidrogenada, pelo fato de se tratar de gordura natural com zero-trans, qualidade muito procurada atualmente pelos fabricantes de gorduras especiais. Além disso, o volume específico dos bolos experimentais, elaborados com gordura de cupuaçu, não foi diferente do bolo padrão, indicando que esta gordura foi capaz de ajudar o glúten na sustentação da estrutura do bolo, propriedade fundamental requerida para uma gordura de panificação. O mesmo autor utilizou a gordura zero-trans e desodorizada de cupuaçu e o liquor de cupuaçu como ingredientes para sorvetes, concluindo que a gordura de cupuaçu pode ser considerada um bom ingrediente na fabricação de sorvetes, com a vantagem de não conter isômeros trans. O sorvete de liquor de cupuaçu foi bem aceito em análise sensorial para consumidor.

\subsection{2 - pH e acidez total titulável}

$\mathrm{Na}$ Tabela 8 apresentam-se os valores de $\mathrm{pH}$ e acidez total titulável das amostras de liquor de cupuaçu.

$\mathrm{O} \mathrm{pH}$ do liquor de cupuaçu ficou na faixa de 5,34 a 5,67. VASCONCELOS [25] obteve para os cotilédones das sementes fermentadas e secas de cupuaçu $\mathrm{pH}$ de 5,34 , valor este considerado dentro da faixa ideal para o cacau fermentado e seco que, segundo ARAGÃO [3], situa-se em torno de 5,0.

TABELA 8. $\mathrm{pH}$ e acidez total titulável das amostras de liquor de cupuaçu.

\begin{tabular}{ccc}
\hline Amostra & $\mathrm{pH}$ & Acidez total titulável (meq NaOH / 100g) \\
\hline LF1 & $5,34 \pm 0,01^{\mathrm{c}}$ & $17,14 \pm 0,22^{\mathrm{a}}$ \\
LF2 & $5,67 \pm 0,03^{\mathrm{a}}$ & $10,75 \pm 0,56^{\mathrm{b}}$ \\
LF3 & $5,57 \pm 0,01^{\mathrm{b}}$ & $10,83 \pm 1,09^{\mathrm{b}}$
\end{tabular}

Os valores de uma mesma coluna, com a mesma letra, não diferem significativamente entre si (Teste de Tukey a $5 \%$ de significância).

As análises de $\mathrm{pH}$ e acidez foram realizadas em triplicata.

LF1 - liquor de cupuaçu proveniente do lote F1 de sementes fermentadas e secas de cupuaçu.

LF2 - liquor de cupuaçu proveniente do lote F2 de sementes fermentadas e secas

LF3 - liquor de cupuaçu proveniente do lote F3 de sementes fermentadas e secas de cupuaçu.

A acidez de LF1 diferiu significativamente de LF2 e LF3. A alta acidez de LF1 pode estar relacionada com o processo fermentativo do lote $\mathrm{F} 1$, do qual foi produzido, pois este não foi satisfatoriamente conduzido. A temperatura da massa de sementes demorou a subir, devido às sementes se encontrarem molhadas pela chuva, retardando a ação dos microrganismos presentes no processo.

Para a amêndoa de cacau, de acordo com ZAMALLOA [26], diversos fatores provocam variações em sua acidez, tais como variedade, maturação do fruto, época de colheita, reião de plantio e, principalmente, na condução do processo fermentativo. Para DIAS [8] a diferença de acidez tem relação com a quantidade de polpa em torno da semente de cacau para a sua fermentação.

De acordo com LOPEZ [18], para a amêndoa de cacau a faixa de acidez desejada pelas indústrias situase entre 12 a $15 \mathrm{meq} \mathrm{NaOH} / 100 \mathrm{~g}$.

\subsection{3 - Análise de cor das amostras de liquor de cupuaçu}

Para a caracterização da cor das amostras de liquor de cupuaçu, utilizou-se o sistema de cores CIELAB com seus três parâmetros: parâmetro $L^{*}$ e as coordenadas cromáticas $\mathrm{a}^{*} \mathrm{e} \mathrm{b}^{*}$. O parâmetro $\mathrm{L}^{*}$ está associado à luminosidade das amostras e pode variar de 0 a 100 , sendo que os valores mais altos de $\mathrm{L}^{*}$ (próximos de 100) caracterizam as amostras mais claras e os menores valores de $L^{*}$ (menores que 50 ) caracterizam amostras mais escuras. A coordenada cromática a* está associada à dimensão verde-vermelho; valores positivos de $a^{*}$ indicam amostras na região do vermelho, valores negativos de $a^{*}$ indicam amostras na região do verde. A coordenada cromática b* está associada à dimensão azulamarelo; valores positivos de $b^{*}$ indicam amostras na região do amarelo, valores negativos de $b^{*}$ indicam amostras na região do azul. 
Na Tabela 9 verificam-se os valores do parâmetro $L^{*}$ e das coordenadas cromáticas $a^{*}$ e $b^{*}$ da análise de cor das amostras de liquor de cupuaçu.

TABELA 9. Valores obtidos do parâmetro $L^{*}$ e das coordenadas cromáticas $\mathrm{a}^{*}$ e $\mathrm{b}^{*}$ da análise de cor das amostras de liquor de cupuaçu.

\begin{tabular}{cccc}
\hline Amostras & L* $^{*}$ & $\mathrm{a}^{*}$ & $\mathrm{~b}^{*}$ \\
\hline LF1 & $42,42^{\mathrm{ab}}$ & $6,50^{\mathrm{b}}$ & $8,62^{\mathrm{b}}$ \\
LF2 & $43,03^{\mathrm{a}}$ & $7,57^{\mathrm{a}}$ & $9,32^{\mathrm{a}}$ \\
LF3 & $42,28^{\mathrm{b}}$ & $7,40^{\mathrm{a}}$ & $8,84^{\mathrm{ab}}$ \\
\hline
\end{tabular}

Os valores de uma mesma coluna, com a mesma letra, não diferem significativamente entre si (Teste de Tukey a $5 \%$ de significância).

LF 1 - liquor de cupuaçu proveniente do lote F1 de sementes fermentadas e secas de cupuaçu.

LF2 - liquor de cupuaçu proveniente do lote F2 de sementes fermentadas e secas de cupuaçu.

or de cupuaçu proveniente do lote F3 de sementes fermentadas e secas de cupuaçu.

Conforme Tabela 9, as amostras de liquor de cupuaçu se apresentaram nas regiões do vermelho e do amarelo, com o valor do parâmetro $L^{*}$ abaixo de $50\left(\mathrm{~L}^{*}<50\right)$, caracterizando como amostras escuras. Observa-se que LF1, cujo processo de fermentação não foi adequadamente conduzido, apresentou valores das coordenadas cromáticas $a^{*}$ e b* inferiores aos de LF2 e LF3, ou seja, sua intensidade de vermelho e de amarelo foi inferior ao das demais amostras.

Alguns fatores podem afetar a coloração do liquor, tais como a fermentação das sementes e o processo de torração [6].

\subsection{4 - Comportamento reológico das amostras de liquor de cupuaçu}

O liquor de cupuaçu, assim como o liquor de cacau e o chocolate, é um fluido não newtoniano, onde o conhecimento de sua viscosidade plástica e limite de escoamento são importantes no dimensionamento de tubulações e sistemas de bombeamento empregados para o seu transporte na planta de processamento. $\mathrm{Na}$ Tabela 10 encontram-se os valores de viscosidade plástica de Casson, limite de escoamento e diâmetro das partículas das amostras de liquor de cupuaçu.

Verifica-se na Tabela 10 que a viscosidade do liquor LF2 foi significativamente superior as demais. Observa-se também que o maior valor de limite de escoamento corresponde ao liquor com menor viscosidade plástica, assim como o menor valor de limite de escoamento corresponde ao liquor de maior viscosidade plástica.

Dentre os principais fatores que afetam a viscosidade plástica de Casson do liquor, tem-se: temperatura, conteúdo de umidade, conteúdo de gordura, tamanho das partículas e adição de lecitina. Quanto maior o conteúdo de umidade e o tamanho das partículas do liquor maior será a sua viscosidade plástica de Casson. Aumentando-se o conteúdo de gordura e a temperatura diminui-se a viscosidade, o mesmo ocorrendo com a adição de lecitina [4, 5, 10, 23]. Neste trabalho o diâmetro das partículas influenciou significativamente a viscosidade das amostras de liquor de cupuaçu, onde para tamanhos maiores de partículas os valores de viscosidade foram mais elevados.

TABELA 10. Viscosidade plástica de Casson (Pa.s), limite de escoamento $(\mathrm{Pa})$ e diâmetro das partículas $(\mathrm{mm})$ das amostras de liquor de cupuaçu.

\begin{tabular}{|c|c|c|c|}
\hline Amostra & $\begin{array}{l}\text { *Viscosidade plástica } \\
\text { Casson (Pa.s) }\end{array}$ & $\begin{array}{c}\text { *Limite de escoamento } \\
(\mathrm{Pa})\end{array}$ & $\begin{array}{l}\text { Diâmetro das partículas } \\
(\mu \mathrm{m}) \text {. Valor cumulativo } \\
(90 \%)\end{array}$ \\
\hline LF1 & $5,04^{c}$ & $0,44^{a}$ & 39,4 \\
\hline LF2 & $15,78^{a}$ & $0,10^{c}$ & 45,8 \\
\hline LF3 & $6,53^{b}$ & $0,39^{b}$ & 40,7 \\
\hline
\end{tabular}

Os valores de uma mesma coluna, com a mesma letra, não diferem significativamente entre si (Teste de Tukey a $5 \%$ de significância).

* Medicões realizadas em triplicata na temperatura de $40^{\circ} \mathrm{C}$.

LF1 - liquor de cupuaçu proveniente do lote F1 de sementes fermentadas e secas de cupuaçu.

LF2 - liquor de cupuaçu proveniente do lote F2 de sementes fermentadas e secas de cupuaçu.

LF3 - liquor de cupuaçu proveniente do lote F3 de sementes fermentadas e secas de cupuaçu.

\section{7 - Resultados das análises físicas e químicas das amostras de gordura de cupuaçu}

Na Tabela 11 apresenta-se a composição percentual de ácidos graxos das amostras de gordura de cupuaçu.

TABELA 11. Composição percentual de ácidos graxos das amostras de gordura de cupuaçu.

\begin{tabular}{lccc}
\hline Ácidos graxos & GF1 & GF2 & GF3 \\
\hline Ácido mirístico (C14:0) & 0,15 & 0,08 & 0,23 \\
Ácido palmítico (C16:0) & 11,70 & 11,25 & 11,22 \\
Ácido palmitoléico (C16:1) & 0,33 & 0,40 & 0,32 \\
Ácido esteárico (C18:0) & 37,86 & 38,09 & 38,15 \\
Ácido oléico (C18:1) & 38,77 & 38,79 & 37,83 \\
Ácido linoléico (C18:2) & 2,37 & 2,39 & 2,44 \\
Ácido aráquico (C20:0) & 7,70 & 7,97 & 7,44 \\
Ácido linoléico (C18:3) & 0,18 & 0,22 & 0,19 \\
Ácido beênico (C22:0) & 0,71 & 0,74 & 0,74 \\
Saturados (\%) & 58,12 & 58,13 & 57,78 \\
Monoinsaturados (\%) & 39,10 & 39,19 & 38,15 \\
Poliinsaturados (\%) & 2,55 & 2,61 & 2,63
\end{tabular}

GF1 - gordura de cupuaçu extraída do liquor de cupuaçu LF1 (proveniente do lote F1 de sementes fermentadas e secas de cupuaçu).

GF2 - gordura de cupuaçu extraída do liquor de cupuaçu LF2 (proveniente do lote F2 de sementes fermentadas e secas de cupuaçu). FF3 - gordura de cupuaçu extraida do liquor de cupuaçu LF3 (proveniente do lote

Dentre os principais ácidos graxos presentes na gordura de cupuaçu, têm-se os ácidos palmítico (C16:0), esteárico (C18:0), oléico (C18:1), linoléico (C18:2) e araquídico (C20:0), totalizando cerca de 97,08 a 98,49\%. A gordura de cupuaçu apresenta significativos teores de ácidos graxos monoinsaturados (38,15 a 39,19\%) e poliinsaturados $(2,55 \%$ a $2,63 \%)$, o que a caracteriza ser uma gordura macia. 
Segundo LUCCAS [19], embora a gordura de cupuaçu apresente ponto de fusão $\left(33,9^{\circ} \mathrm{C}\right)$ superior ao da manteiga de cacau $\left(31,0^{\circ} \mathrm{C}\right)$, o seu alto teor de ácidos graxos monoinsaturados, principalmente o ácido oléico, torna esta gordura mais macia.

Na Tabela 12 apresenta-se a composição de triacilgliceróis das amostras de gordura de cupuaçu.

TABELA 12. Composição de triacilgliceróis das amostras de gordura de cupuaçu.

\begin{tabular}{|c|c|c|c|c|}
\hline \multicolumn{2}{|c|}{ Triacilgliceróis } & \multirow{2}{*}{$\begin{array}{c}\text { GF1 } \\
-\end{array}$} & \multirow{2}{*}{$\begin{array}{c}\text { GF2 } \\
-\end{array}$} & \multirow{2}{*}{$\begin{array}{c}\text { GF3 } \\
-\end{array}$} \\
\hline PliP & P-Palmitico & & & \\
\hline O०० & S-Esteárico & 2,65 & 2,58 & 2,82 \\
\hline $\mathrm{POO}$ & O-Oléico & 4,06 & 3,98 & 4,65 \\
\hline Plis & Li-Linoléico & 0,94 & 0,80 & 1,17 \\
\hline POP & A-Araquidico & 1,03 & 0,95 & 1,16 \\
\hline SOO & & 15,98 & 16,38 & 17,41 \\
\hline POS & & 11,64 & 11,87 & 11,65 \\
\hline OOA & & 9,13 & 9,01 & 8,76 \\
\hline sos & & 28,87 & 30,18 & 30,43 \\
\hline PSS & & 1,61 & 1,50 & 1,64 \\
\hline SOA & & 17,63 & 18,24 & 17,23 \\
\hline OAA & & 4,12 & 3,86 & 2,94 \\
\hline Outros & & 1,34 & 0,65 & 0,14 \\
\hline SUS & S-Saturado & 60,11 & 62,04 & 61,64 \\
\hline SSS & U - Insaturado & 1,61 & 1,50 & 1,64 \\
\hline SUU & & 20,04 & 20,36 & 22,06 \\
\hline UUU & & 2,65 & 2,58 & 2,82 \\
\hline UUS & & 9,13 & 9,01 & 8,76 \\
\hline USS & & 4,12 & 3,86 & 2,94 \\
\hline
\end{tabular}

GF1 - gordura de cupuaçu extraída do liquor de cupuaçu LF1 (proveniente do lote F1 de sementes fermentadas e secas de cupuaçu).

GF2 - gordura de cupuaçu extraída do liquor de cupuaçu LF2 (proveniente do lote F2 de sementes fermentadas e secas de cupuaçu). FF3 - gordura de cupuaçu extraída do liquor de cupuaçu LF3 (proveniente do lote

Segundo LUCCAS [19], os triacilgliceróis SOO, OOA e OOO da gordura de cupuaçu também podem ser os responsáveis pela sua maciez, enquanto que os triacilgliceróis SOA e OAA podem explicar seu maior ponto de fusão comparado ao da manteiga de cacau.

A gordura de cupuaçu apresenta alto teor de triacilgliceróis simétricos, do tipo SUS (saturado, insaturado, saturado). De acordo com LUCCAS [19], esse alto teor de triacilgliceróis simétricos pode indicar que a gordura de cupuaçu possui características de cristalização e polimorfismo próximos aos da manteiga de cacau. O polimorfismo resulta dos diferentes modos possiveis de empacotamento molecular do cristal. A forma polimórfica está normalmente relacionada à composição triglicerídica da gordura, o que irá influenciar na aparência ou textura da gordura e exercer influência durante o processamento.

Em estudos realizados por LUCCAS [19], o autor concluiu que, embora apresente algumas semelhanças com a manteiga de cacau, de acordo com a EU (União
Européia), a gordura de cupuaçu não pode ser considerada como gordura equivalente à manteiga de cacau, ou $\mathrm{CBE}$, pois não satisfaz a dois critérios analíticos estabelecidos: possui menos que $65 \%$ de triacilgliceróis do tipo SOS (saturado, ácido oléico, saturado) e apresenta mais que $45 \%$ de ácidos graxos insaturados. O autor detectou em gordura de cupuaçu cerca de $29,13 \%$ de SOS e $45,7 \%$ de ácidos graxos insaturados. No entanto, a gordura de cupuaçu pode ser utilizada com sucesso na fabricação de chocolates amargo, ao leite e branco, contribuindo com até $5 \%$ com base no peso total da formulação, sem alterar as características físicas e sensoriais do produto.

LANNES, MEDEIROS \& GIOIELL [16] analisaram as propriedades físicas e a cristalização da gordura de cupuaçu e da manteiga de cacau e a mistura das duas, e concluíram que a gordura de cupuaçu apresenta dureza menor e tempo de cristalização inferior ao da manteiga de cacau. O conteúdo de gordura sólida, a temperatura ambiente, da manteiga de cacau é superior ao da gordura de cupuaçu e da mistura. Segundo os referidos autores, a gordura de cupuaçu e a sua mistura com a manteiga de cacau podem ser usadas na elaboração de produtos análogos ao chocolate para localidades cuja temperatura são amenas.

A substituição total da manteiga de cacau por gordura de cupuaçu na formulação do chocolate, assim como a de liquor de cacau por liquor de cupuaçu proporciona produto de maior maciez, devido à composição química da gordura de cupuaçu. Tal fato foi comprovado por COHEN [6], onde, ao elaborar um produto análogo de chocolate ao leite com 100\% de substituição de liquor e gordura de cupuaçu, obteve força de ruptura de $1,78 \mathrm{kgf}$ contra 4,40kgf do chocolate. Essa maciez foi bastante apreciada por consumidores no teste de aceitação.

Na Tabela 13 apresentam-se os valores de viscosidade das amostras de gordura de cupuaçu.

TABELA 13. Viscosidade das amostras de gordura de cupuaçu.

\begin{tabular}{cc}
\hline Amostra & *Viscosidade (Pa.s) \\
\hline GF1 & $39,53 \times 10^{-3}$ \\
GF2 & $40,43 \times 10^{-3}$ \\
GF3 & $39,77 \times 10^{-3}$
\end{tabular}

*Medições realizadas na temperatura de $40^{\circ} \mathrm{C}$

GF1 - gordura de cupuaçu extraída do liquor de cupuaçu LF1 (proveniente do lote F1 de sementes fermentadas e secas de cupuaçu).

GF2 - gordura de cupuaçu extraída do liquor de cupuaçu LF2 (proveniente do lote F2 de sementes fermentadas e secas de cupuacul.

GF3 - gordura de cupuaçu extraída do liquor de cupuaçu LF3 (proveniente do lote F3 de sementes fermentadas e secas de cupuaçu).

Quanto a reologia da gordura de cupuaçu, verificou-se que a $40^{\circ} \mathrm{C}$ esta apresenta comportamento newtoniano.

\section{4 - CONCLUSÕES}

As etapas de processamento (fermentação, secagem, descascamento, torração, moagem e refino) apli- 
cadas neste trabalho são adequadas para a obtenção de liquor de cupuaçu de qualidade, apresentando este aroma e aparência característicos e semelhantes ao liquor de cacau. Sua principal característica é a presença de alto teor de gordura (63,93 a 66,51\%), podendo esta ser aplicada nas indústrias alimentícia e farmacêutica.

\section{5 - REFERÊNCIAS BIBLIOGRÁFICAS}

[1] AOAC. Official methods of analysis of the Association of Official Analytical Chemists: edited Ig W. Horwitz $16^{a}$ ed. Washington, 850p. v.2. 1997

[2] AOCS. Official methods and recommended practices of the American Oils Chemist Society. 3 ed. Champaign. v.1-2. 1993.

[3] ARAGÃO, C.G. Mudanças físicas e quimicas da semente do cupuaçu (Theobroma grandiflorum Schum.) durante o processo fermentativo. 1992, 115p. Tese (Mestrado) - Fundação Universidade do Amazonas.

[4] CHEVAlley, J. Rheology of chocolate. Journal of Texture Studies, v.6, n.1, p.177-196, Boston, Jan., 1974.

[5] CHEVAlley, J. Chocolate flow properties. In: Industrial Chocolate Manufacture and Use. Editado por Beckett, 2.ed. England: Clays Ltd., p.139-155, 1994.

[6] COHEN, K.C. Estudo do processo de temperagem do chocolate ao leite e de produtos análogos elaborados com liquor e gordura de cupuaçu. 2003, 296p. Tese (Doutorado em Tecnologia de Alimentos) - Faculdade de Engenharia de Alimentos, UNICAMP.

[7] CONCEX, Conselho Nacional do Comércio Exterior. Resolução $\mathrm{n}^{\circ} 42$. Rio de Janeiro. 9p. 1968. Brasil.

[8] DIAS, J. C. Permeabilidade da casca da semente de cacau ao ácido acético: evolução na fermentação e efeito da adição de celulases, antes da secagem, na acidez do produto final. 1987, 70p. Tese (Mestrado) - Escola Superior de Lavras, ESAL.

[9] FANG, T.N.; TIU, C.; WU, X. \& DONG, S. Rheological behaviour of cocoa dispersions. Journal of Texture Studies, v.26, p.203-215, 1995.

[10] GILABERT-ESCRIVÁ,M.V. Comparação das propriedades reológicas da massa de cacau torrada convencionalmente e por microondas. Campinas, 1997. 88p. Dissertação (Mestrado) - Faculdade de Engenharia de Alimentos - Universidade Estadual de Campinas (UNICAMP).

[11] GILABERT-ESCRIVÁ,M.V. Caracterização e seleção de gorduras do gênero Theobroma para aplicação tecnológica. 2002, 165p. Tese (Doutorado em Tecnologia de Alimentos) - Faculdade de Engenharia de Alimentos, UNICAMP.
[12] GOERING, H. K. \& VAN SOEST, P. J. Forage fibre analysis. Agri-handbook: Agriculture Research Service, U. S. Dept. Agriculture, 1970, 375p.

[13] GRIMALDI, J. Les possibilités D‘amélioration des techniques D'ecabossage et de fermentation dans le processus artisanal de la préparation du cacao. Café, Cacao, Thé, v.22, p.306-316, 1978.

[14] HARTMAN, L.; LAGO, R. Rapid preparation of fatty acid methyl esters from lipids. Lab. Pract. London, v.22, p.475-476, 1973.

[15] KALIL, A. C. Manual Básico de Nutrição. São Paulo: Instituto de Saúde, 1975.

[16] LANNES, S.C.S.; MEDEIROS, M.L.; GIOIELLI, L.A. Interacciones fisicas entre grasas de cupuassu y cação. Grasas y Aceites, v.54, n.3, p.253-258, 2003.

[17] LOPES, A.S; GARCÍA, N.H.P.; VASCONCELOS, M.A.M. Avaliação das condições de torração após a fermentação de amêndoas de cupuaçu (Theobroma grandiflorum Schum.) e cacau (Theobroma cacao L.). Braz. J. Food Technol, v.6, n.2, p.309-316, 2003.

[18] LOPEZ, A.S.F. Factors associated with cocoa bean acidity and the possibility of its reduction. Revista Theobroma (Brasil), v.13, n.3, p.233-248, 1983.

[19] LUCCAS, V. Fracionamento térmico e obtenção de gorduras de cupuaçu alternativas à manteiga de cacau para uso na fabricação de chocolate. 2001, 195p. Tese (Doutorado em Engenharia Química). Faculdade de Engenharia Quimica, UNICAMP.

[20] PASSMORE, R.; NICOL, B. M. \& RAO, M. N. Manual sobre Necessidades Nutricionales del Hombre. Ginebre: OMS, 1975.

[21] PEREIRA FILHO, J. Os bons frutos do Norte e Nordeste. Gazeta Mercantil, São Paulo, 14 fev, p.8, 2001.

[22] QUESNEL, V.C. \& LOPEZ, A. A sweat-box for fermentation small samples of cacao. Tropical Agriculture, Trinidad, v.4, n.52, p.309-316, 1975.

[23] TANERI, C.E. Chocolate Liquors: Factors Affecting Viscosity. The Manufacturing Confectioner, March, 1976, p. 45-55.

[24] UNITED STATES DEPARTAMENT OF AGRICULTURE. Composition of Foods. Washington: USDA, 1963.

[25] VASCONCELOS, M.A.M. Transformações físicas e químicas durante a fermentação de amêndoas do cupuaçu (Theobroma grandiflorum Schum). 1999, 114 p. Tese (Mestrado em Tecnologia de Alimentos) Faculdade de Engenharia de Alimentos, UNICAMP.

[26] ZAMALLOA, C.W.A. Caracterização físico-química e avaliação de metil pirazinas no desenvolvimento do sabor, em dez cultivares de cacau (Theobroma cacao L) produzidos no Estado de São Paulo. 1994, 121 p. Tese (Doutorado em Tecnologia de Alimentos) Faculdade de Engenharia de Alimentos, UNICAMP. 